\title{
Nanowire Joining Methods
}

\author{
Xiaopeng Li, Fan Gao, and Zhiyong $\mathrm{Gu}^{*}$ \\ Department of Chemical Engineering, University of Massachusetts Lowell, One University Avenue, Lowell, MA 01854, \\ USA
}

\begin{abstract}
Recent advances in nanowire research emphasize on its promising applications in nanoelectronics, nanophotonics, nanomedicine, and nanoelectromechanical systems (NEMS). Even with many advances, the joining of nanowires has become a critical issue for future device miniaturization and integration. With the help of in situ manipulation, directed assembly and self-assembly, much progress has been made towards joining of nanowires such as welding, soldering, fastener materials, and controlled localized deposition. These methods provide multiple choices for joining nanowires of various materials, at the mean time good electrical conductivity and mechanical strength can be achieved. This paper reviewed the state-of-the-art techniques for joining nanowires, and discussed the advantages and limitations of each method. The process and mechanism of these techniques are described in three categories: nanowirenanowire joining, nanowire network joining, and nanowire-contact pad/electrode joining.
\end{abstract}

Keywords: Nanowire, joining, welding, soldering, assembly, nanoelectronics, NEMS.

\section{INTRODUCTION}

Ever since Iijima discovered the carbon nanotubes in 1991 [1], the heat of nano related researches and industrial development has spread all over the world and tremendous effort has been made in the synthesis of novel nanomaterials and nanostructures. Now, after two decades of progress, numerous nanomaterials have been successfully fabricated and their structures and properties intensively studied. Among them, one-dimensional nanostructures, especially nanowires have received substantial interest due to their outstanding electrical, optical, magnetic and biological properties. In the field of electronic engineering, wire-shaped objects are obviously the most used components, because of their geometrical efficiency in electron transportation and building connections to form compact circuits. Hence nanowires, without doubt, play a significant role in the progress of device miniaturization toward micro/nano electronics and devices. Plus, nanowires are capable of being used as functional components other than just electrical connection and mechanical support, e.g. semiconducting nanowires for field effect transistors (FETs) [2], surface modified nanowires for bio-detection [3]. So far, researchers have gained much experience in fabricating nanowires using physical deposition (vapor-solid/vapor-liquid-solid/solutionliquid-solid growth) [4-6], electrospinning [7], electrodeposition $[8,9]$, thermal oxidation growth $[10,11]$ and nanolithography [12,13]. Up to date, metal [14], semiconductor [15] and conducting polymer [16] have been utilized to make nanowires. This large variety in composition enables the promising applications of nanowires as transistor [17, 18], chemical sensor [19, 20], optics [21], catalyst [22, 23], battery [24, 25], solar cells [26, 27], etc.

*Address correspondence to this author at the Department of Chemical Engineering, University of Massachusetts Lowell, USA; Tel: 1-978-9343540; E-mail: Zhiyong_Gu@uml.edu
Nowadays, more attention is being paid to some new fields which make use of and benefit from nanowires, including photonics $[28,29]$, optoelectronics $[21,30]$ and NEMS [31, 32]. Self-assembly [33, 34] and directed assembly $[35,36]$ of nanowires have been achieved with the help of molecular interactions and micro/nano fabrication [37]. However, there are still several technical obstacles against fully use of the unique properties of nanowires, for example, some assembled nanowires are loosely connected to each other or to the electrodes/contact pads without effective interconnections. This would bring up the issue for effectively joining nanowires.

Various joining processes such as welding, soldering and mechanical bonding are being developed for the formation of nanowire interconnects, networks and contacts with electrodes. This review article summaries the recent progress in nanowire joining, and presents the categorized joining methods by three formations mentioned above. Specific joining mechanism and features of each method are discussed to provide a better understanding of joining in the nano-scale.

\section{CURRENT STATE-OF-THE-ART JOINING METHODS}

Confined by the current shaping and machining techniques $[38,39]$, some of the widely used joining methods are not applicable in nanoscale. For example, nanoscale mechanical bonding methods using seals and gaskets are not available currently since they require the fabrication of objects even smaller than the structures of research interest. Also, for mechanical joining systems, screws and bolts are commonly used yet cheap and easy to deploy the joining; however, unless there is much progress on micro machining equipments, it is not possible to do machining on nano-objects which probably need the precision around sub angstrom level. Meanwhile, some delightful aspects of nanostructures such as melting point depression, ultra pure and smooth surface, have illustrated 
that there is much room to develop new joining methods based on these unique properties that only exist in this small size scale. For example, Lu et al. demonstrated cold joining of ultra-thin gold nanowires that occurs at room temperature in vacuum [40]; $\mathrm{Gu}$ et al. explored the use of diffusion bonding to form metallic contacts between gold nanowires in liquid phase at a temperature range much lower than the melting point of gold [41]. These could make simple and spontaneous joining process to compensate the inability of being machined.

In Table 1, we listed the current methods for nanowire joining, including their advantages and limitations. The following context on nanowire joining will be divided into three sections - nanowire-nanowire joining, nanowire network joining and nanowire-contact pad/electrode joining, according to the type of contact formation. Detailed discussion of each method will be presented.

\section{NANOWIRE-NANOWIRE JOINING}

\subsection{Cold Welding}

Cold welding of nanowires could be considered as an excellent example to illustrate the significant difference on material properties when its size reduced from macroscale to nanoscale. Lu et al. have shown that nearly perfect joining was achieved between two gold nanowires or silver nanowires by cold-welding technique. High-resolution transmission electron microscopy (HRTEM) and in situ measurements further revealed that the welded regions have the same crystal orientation, strength and electrical conductivity as the rest of the nanowire [40]. In their experiments, only mechanical contact alone could trigger the welding process in high vacuum at room temperature, and it took a very short time period to complete the welding process. In conventional cold welding, a high pressure is applied to flatten surface, lowering the asperity and making more touching points, while in nanowire cold welding this is not necessary since in nanoscale the contact condition is more ideal. Also, gold is well known to be a malleable and ductile metal with a good resistance to oxidation and cold welding of gold under ambient conditions without high loading has been studied by Ferguson et al. [42]. Noticeably, the welding of two single nanowires can be carried out in both head-to-head and side-to-side mode. Fig. (1) shows the welding process of two gold nanowires imaged by HRTEM, from indicating (a) head-to-head and (b) side-to-side joints formed by cold welding process. Atomic diffusion and surface relaxation play an important role in both macro and micro scale cold welding [42-44]. Also, an 'orientedattachment' mechanism as reported for PdSe nanocrystal was mentioned by author to explain the matching of nanowires' crystallinity [45, 46].

\subsection{Fusion Welding}

Fusion welding is a process that involves the formation and solidification of a liquid phase at the interface. Conventional arc welding, laser welding and electron beam welding all belong to this category. Fusion welding in the nano-scale could be accomplished in several ways, such as Joule heating, electron-induced local heating, or direct heating. All of these methods have been proved to cause the chain action of phase change $\rightarrow$ inter-diffusion $\rightarrow$ cooling down, to join similar or dissimilar metal/semiconductor materials.

Tohmyoh and colleagues [47] used nano-manipulators to place two platinum nanowires in contact, and then applied a constant direct current to perform the welding. Fig. (2) shows the scanning electron microscopy (SEM) images of two platinum nanowires after straight welding (a) and lap welding (b). From the images, the typical morphological deformation was observed. The underlying mechanism is that once two nanowires were brought into contact, the real touching area is quite small. The resulted contact resistance is very high compared with that of nanowire due to the slightly irregular surface of the nanowires. When electrical current was applied, this specific region would accumulate a

Table 1. Current Nanowire Joining Methods

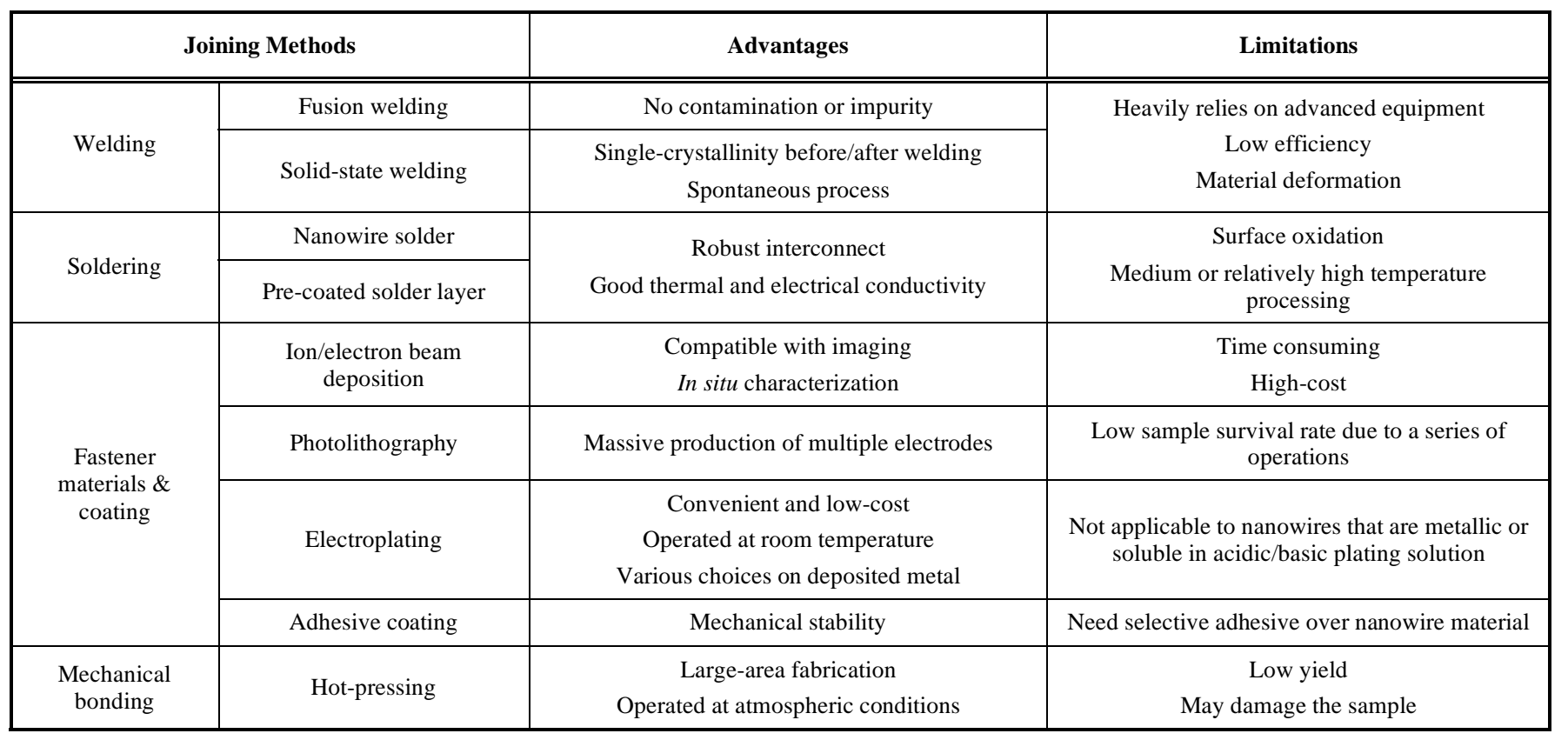




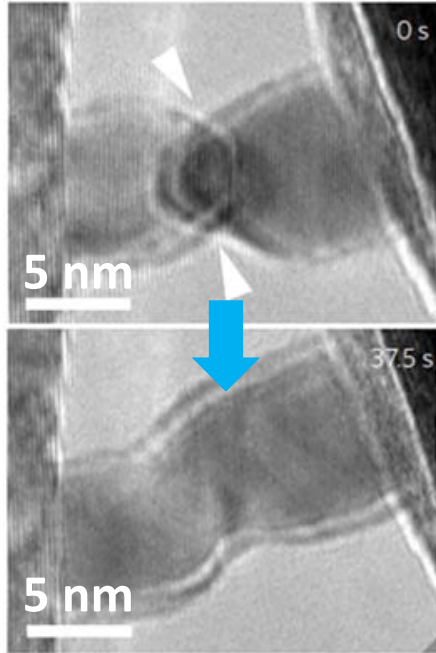

(a)
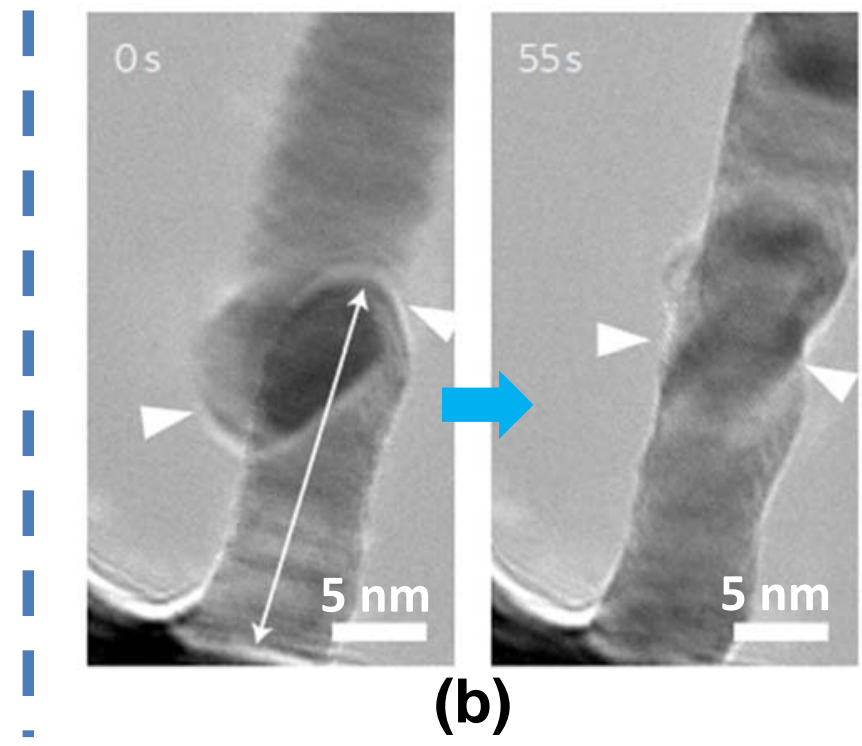

Fig. (1). (a) Head-to-head. (b) Side-to-side welding of two ultrathin gold nanowires. Reprinted with permission [40].

large amount of heat by Joule heating to locally rise the temperature high enough to melt the metal. Once the nanowire tips melted locally to form better contact, both electron and thermal conduction would get prompted, leading to heat generation drop and the heat conduction in the nanowires, respectively. Counting in the heat convection from the surfaces, the heat lost forced the temperature at the contact area fell rapidly as the metal solidified, and the weld phenomenon occurred. Recent progress in the technique of welding by Joule heating is reported by Fukui and colleagues [48]. Using the same concept and repeated welding procedure, Pt nanowires have been assembled into T-shaped (2D) and triaxis-shaped (3D) structures.

Fusion welding of nanowires can also be conducted using electron beam as the heating source. Although traditionally electron beam is mainly used in electron microscopy for material characterization, providing topography images and compositional analysis, several new applications of electron beam have been developed for lithography, restructuring, metallization and heat source. When the electron beam hit a solid nanowire, electrons tend to lose a small portion of the kinetic energy via inelastic scattering within the specimen. These inelastic events cause heat generation, and the consequential effects at atomic level include diffusion and heat dissipation, etc. [49-53]. So if the electron beam was targeted at the junction area of two nanowires, the irradiated local melting could be utilized for the welding process. Fig. (3) shows the welding results of single-crystalline gold nanowires being welded at their overlapping regions to from a closed loop [54]. Besides gold nanowires, silver, copper, nickel, tin and silicon nanowires were also welded using the same method. The successful welding of dissimilar materials further proved the magnificent heat generated by electron beam.

Melting is critical in fusion welding process. If certain confinement could be applied to surround the molten materials like a mold, the joining parts after solidification would still keep the original wire shape. In particular, $\mathrm{Wu}$ and Yang reported a method of joining two germanium nanowires sealed inside carbon sheath [55]. Germanium nanowires were first synthesized via vapor-liquid-solid mechanism, and then coated with a thin layer of carbon sheath which has a high melting point and could provide mechanical support when germanium melts. When two individual nanowires were manipulated into contact, the sheath ruptured at the tips. If the sample stage further heated the inside germanium nanowires over melting temperature, the liquid germanium would spray out and eventually merge to a single wire as shown in Fig. (4).
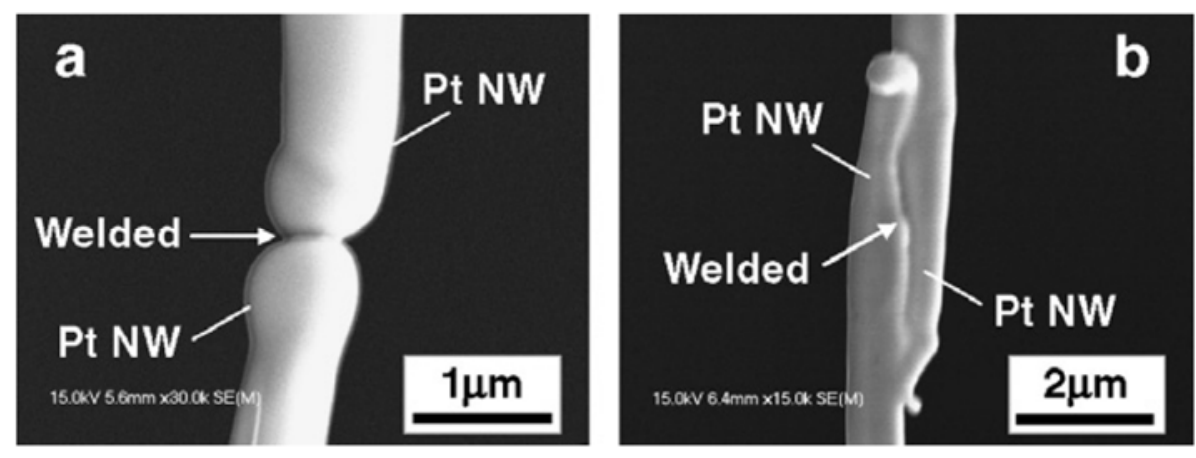

Fig. (2). SEM images of welded platinum nanowire. (a) Straight welding. (b) Lap welding. Reprinted with permission [47]. 


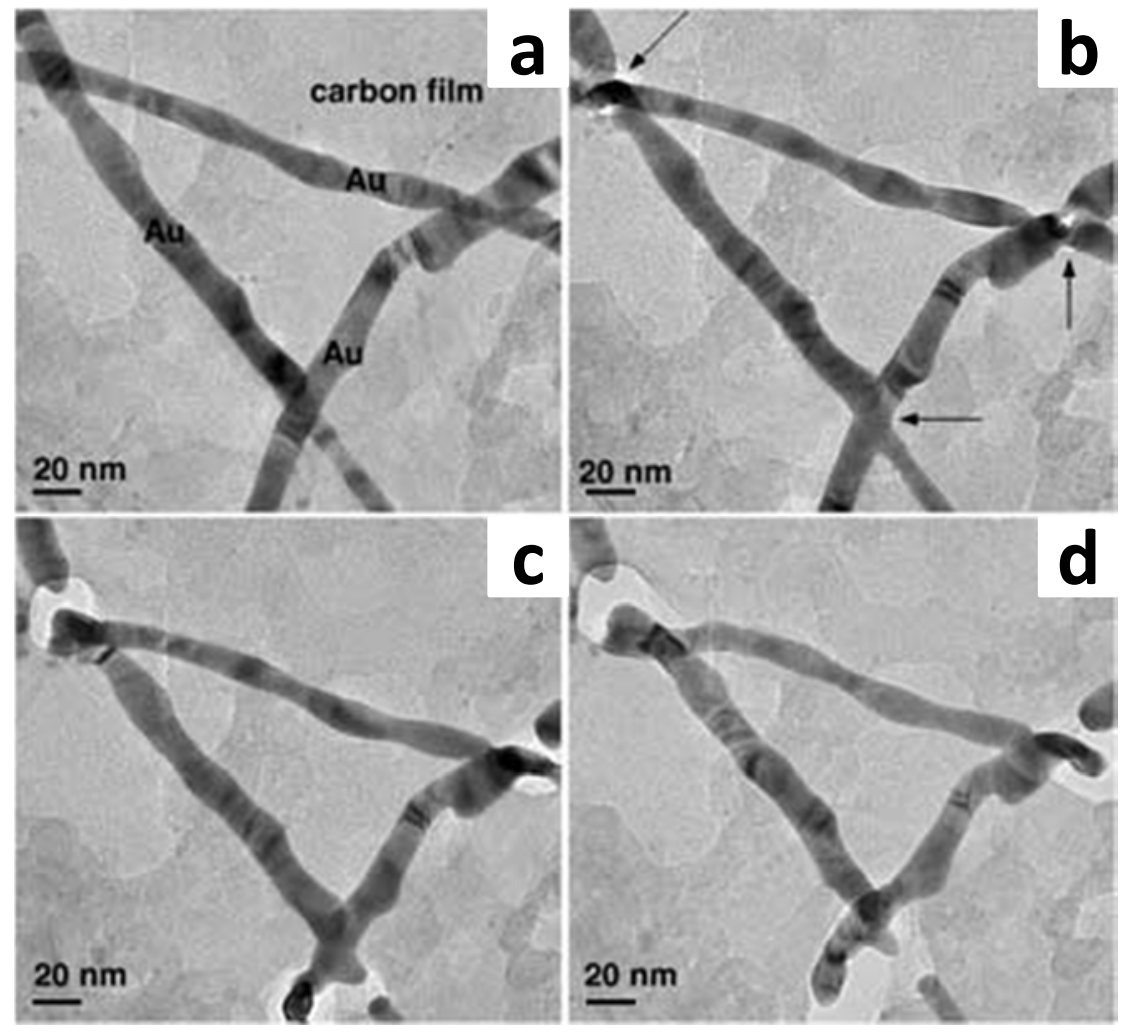

Fig. (3). Welding of three single-crystalline Au NWs with a 10-nm diameter by a high-intense electron beam (HIEB). (a) The original Au NWs. (b-d) A closed loop is formed after welding the NWs together at their overlapping regions (highlighted by arrows), and then the loop is cut off from the rest of the NWs. Holes are seen in the supporting carbon film under the joints. The whole process from (a) to (d) takes 30 min. Reprinted with permission [54].

\subsection{Soldering}

The successful use of nanoscale solder materials to assemble nanowires has been demonstrated by several researches. Peng and colleagues [56] showed that two individual nanowires were selected and positioned by SEM nanomanipulators into a desired pattern; a sacrificial nanowire was picked up by the same manipulators at two
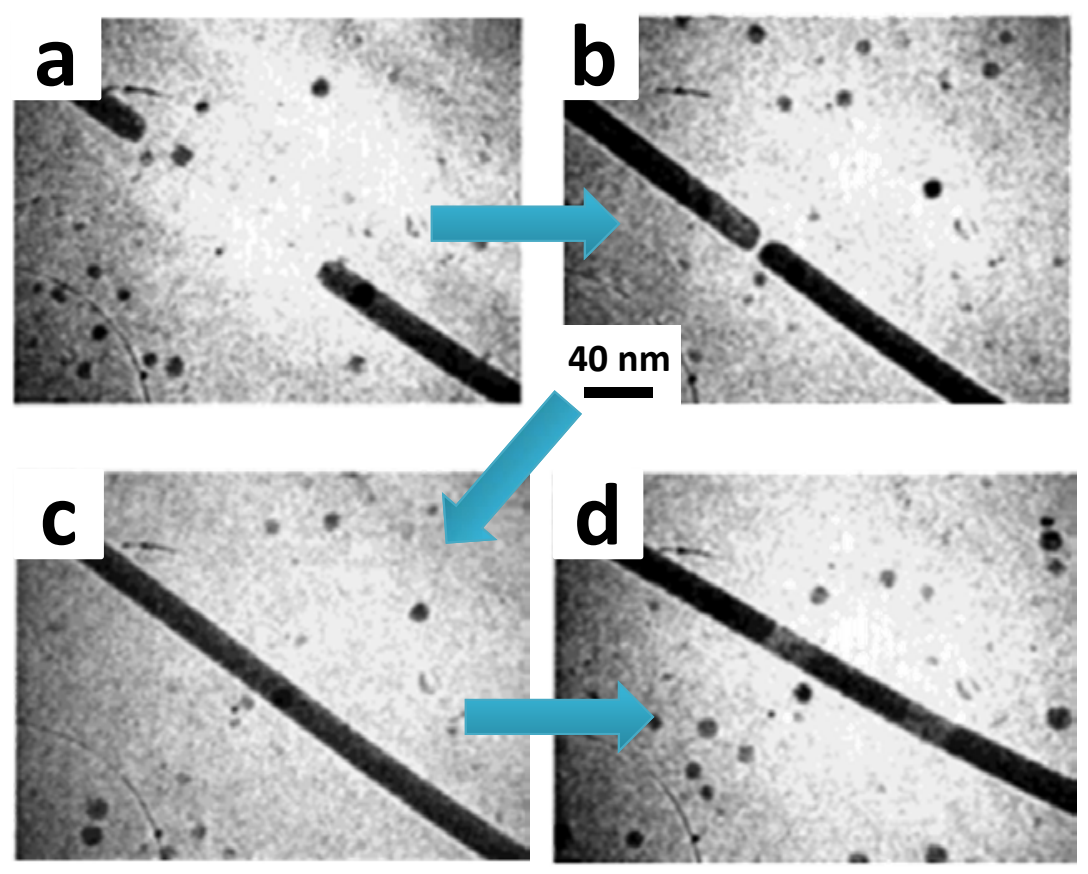

Fig. (4). The TEM image sequence of linking two nanowires within a nanotube. The temperature is kept at $850{ }^{\circ} \mathrm{C}$ during the operation (a-c). After interconnection, the wire is quenched and recrystallized at $540{ }^{\circ} \mathrm{C}(\mathbf{d})$. Reprinted with permission [55]. 
ends (Fig. 5a), and later on to be placed in the junction area to perform soldering (Fig. 5b-d). The good electrical conductivity of this solder joint was observed by testing the system resistance at a wide range of input voltages. Unlike fusion welding, the softening or melting only occurs to the solder materials in soldering process, though there is slight diffusion between solder material and nanowires on some level. As mentioned in reference [56], a current was applied for 1 2 min in order to pre-heat and soften the sacrificial solder nanowire by Joule heating. A rectangular voltage pulse was then applied when the solder nanowire made contact with target objects, and on this step the Joule heating was increased to cause melting and diffusion. Since the solder nanowire could be tin, gold and tin-gold alloy [57], the amplitude of current and voltage pulse applied for different materials may vary.

Another approach to realize nanowire soldering is to fabricate nanowires of low melting point materials and simply conduct the reflow process as in conventional electronics integration. Thanks to the development of nanowire synthesis techniques, various solder materials like indium, tin, indium-tin, and tin-lead alloy $[14,16,58]$ have been fabricated as nanowires. Fig. (6a) shows two nanowires joined by a solder joint to implement this concept [59]. In this case, solder materials were fabricated as a cap segment at one end of nanowires, thus the solder process occurred only at the interconnection area. Further investigation on the joining region (Fig. 6b) showed the presence of oxygen after solder reflow due to the oxidation of materials at atmospheric conditions. The reflow and electrical characteristics of this type of nanowire solder was systematically studied by $\mathrm{Gu}$ and colleagues [58].

\subsection{Adhesive Coating}

Polymer adhesive coating has been used for joining multisegmented nanowires such as $\mathrm{Au}-\mathrm{Ni}-\mathrm{Au}$ nanowires [58]. Before assembly, the nanowires were immersed in a hydrophobic thiol (hexadecane thiol/HDT) solution (in ethanol). Due to the HDT bonding preference on gold over nickel/surface oxide, two kinds of metal segments were selectively patterned to be either hydrophobic (gold segment) or hydrophilic (nickel segment). In a suspension system, the hydrophobic adhesive preferentially precipitated on the hydrophobic gold segments, leading to the aggregation of nanowires on agitation. The adhesive could also be polymerized using ultraviolet light or heat to harden the adhesive and strengthen the joint. The secondary electron and backscattered electron image of two nanowires joined at the gold tips by adhesive were shown in Fig. (7) [60].

\section{NANOWIRE NETWORK JOINING}

\subsection{Welding}

The welding of nanowire network is mainly utilized for two-dimensional (2D) and three-dimensional (3D) integration for miniature electronic devices, such as integrated circuits, CMOS circuits. It usually involves the joining of nanowires in a large quantity, and the final 3D or 2D structure can be fabricated using either nanowire arrays or individual nanowires. In Fig. (8), two 2D copper nanowire arrays attached on silicon substrates were placed in face-toface contact at $300{ }^{\circ} \mathrm{C}$ with the assistance of an applied pressure of 1.3 MPa [61]. After 1 hour of bonding, some nanowires with good vertical alignment were welded together while the rest were misaligned and still separated. This illustrates that for nanosized objects even a small amount of heat and compression could initiate the welding.
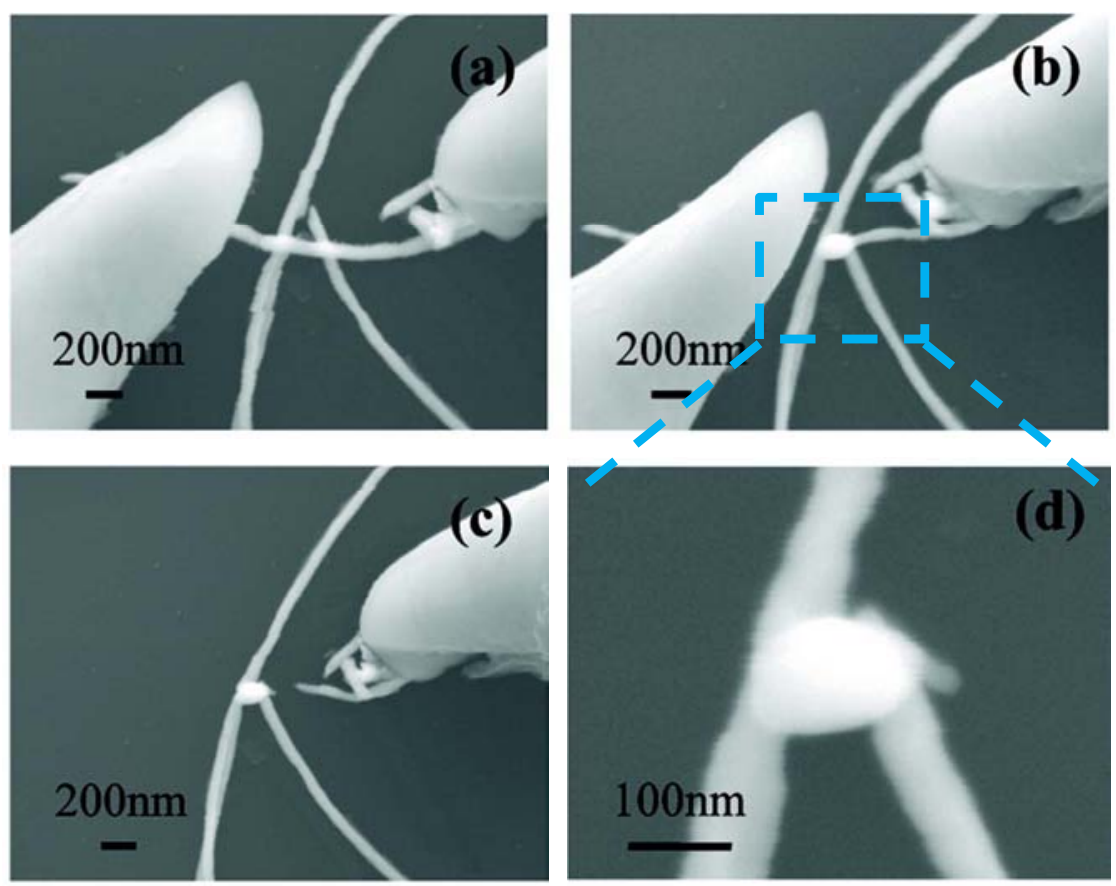

Fig. (5). Nanosoldering gold nanowire structures using sacrificial solder nanowire. (a) Softening of the solder nanowire and contact to the two nanowires to be soldered. (b) Nanosoldering two nanowires together by a rectangular voltage pulse through the solder nanowire, causing material diffusion onto the junction. (c) Mechanically removal of the solder nanowire, leaving miniaturized pattern. (d) High magnification SEM image of the solder joint. Reprinted with permission [56]. 

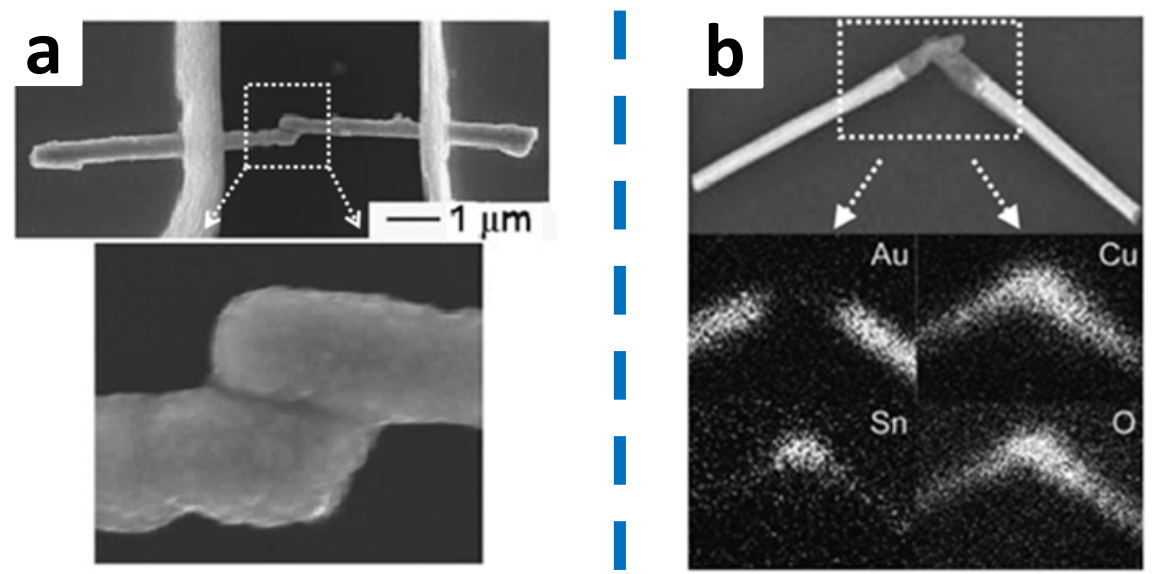

Fig. (6). (a) SEM image of contact pads patterned on top of two soldered nanowires and a zoom-in image of the solder joint [59]. (b) Scanning-electron microscopy-EDS maps of an oxidized joint at atmospheric conditions after solder reflow, between $\mathrm{Au}-\mathrm{Cu}-\mathrm{Sn} / \mathrm{Pb}$ nanowires showing the presence of oxygen in the joint region [58].

Another interesting flash welding phenomenon was reported by Thomson et al., featuring the use of metal chalcogenides nanowires, such as $\mathrm{Bi}_{2} \mathrm{~S}_{3}$, and single-walled carbon nanotubes (SWCNT) [62]. These two materials were first made into thin films and then attached together. The $\mathrm{Bi}_{2} \mathrm{~S}_{3}$ nanowire layer was put on top to ensure that during exposure there was certain amount of light transmitting to reach the interface with the SWCNT layer. Due to the strong adsorbing and photothermal response under light irradiation [63-66], SWCNTs could induce local heating and further melt the $\mathrm{Bi}_{2} \mathrm{~S}_{3}$ nanowire close to the interface to form continuous films.
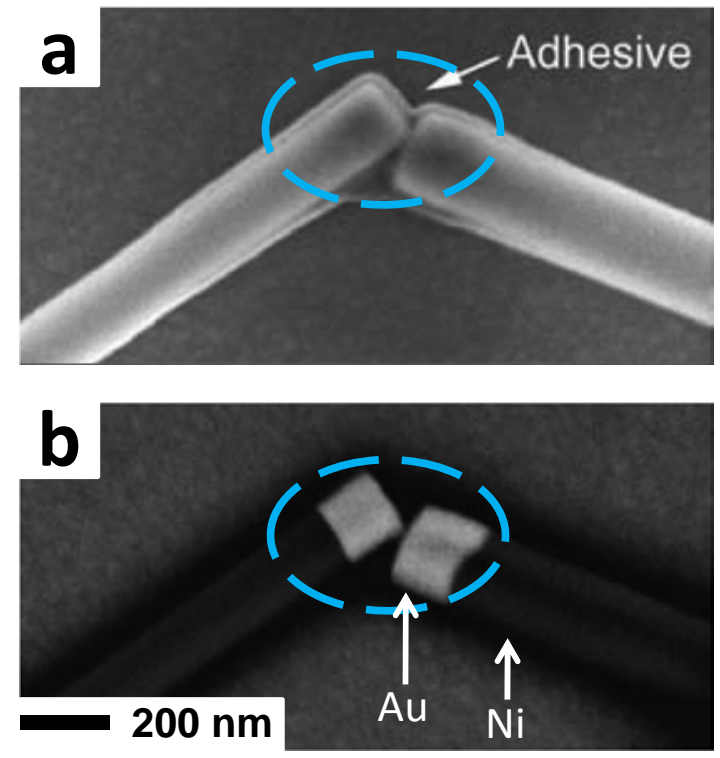

Fig. (7). SEM images showing two nanowires held together by the polymerized adhesive. (a) Secondary electron image showing the polymeric adhesive and the nanowires. (b) Backscattered electron image (brighter regions correspond to materials with higher atomic numbers). The polymer is not visible; the Au segments appear brighter than the $\mathrm{Ni}$ ones [60].

\subsection{Diffusion Bonding}

Although similar morphological deformation as fusion welding could be observed, diffusion bonding is still considered a type of solid state welding process, mainly because it can occur at a temperature range only about $25 \%$ of the melting point of the bulk material. $\mathrm{Gu}$ and colleagues have used an acidic solvent as the medium to carry out the bonding process in nanowire systems [41]. A comparison between gold nanowires before (Fig. 9a) and after (Fig. 9b) bonding is shown with the details of nanowire interconnects and the 3D network structure shown in Fig. (9c) consists of large number of pure diffusion bonded nanowires. The following electrical measurement of the bonded 3D nanowire network indicates that it is electrically conductive and has a low resistance at tens of ohm level, indicating that it is possible to enable 3D spatial sensing or surface related applications such as catalysts [41, 67]. Other than welding metallic nanowires, this method could also be extended to form ohmic contact with low contact resistance between metal-semiconductor/ceramic interfaces [68-70].

\subsection{Soldering}

The most distinguished features of nanowire soldering involve the use of lead-free solder materials and solder on multisegmented nanowires. As well known, the use of lead alloy solder materials in electronics industry has raised much concern due to environmental, health and safety issues worldwide [71-73]. The promising alternatives of tin-lead solders have been more and more studied such as tin, tinsilver and tin-silver-copper (SAC) alloys with low melting point. The research work of using lead-free nanosolders for nanowire integration has been devoted to studying soldering techniques applied in nanowires joining and assembly [74, 75]. To realize nanoscale soldering, these materials were fabricated into solder segments caped on both ends of a single nanowire by the well-established electrodeposition method. This unique structure was able to join the nanowires closely contacted with each other where the joining would only take place at solder covered area, and thus the unnecessary interconnects with functional segments were avoided. Comparing to the expensive and time-consuming joining, these interconnects between nanowires formed by the soldering technique are relatively easy to perform and do not rely on advanced equipments. A large scale 3D nanowire network has been assembled by a "liquid reflow" process with external magnetic field assistance [75]. The traditional 

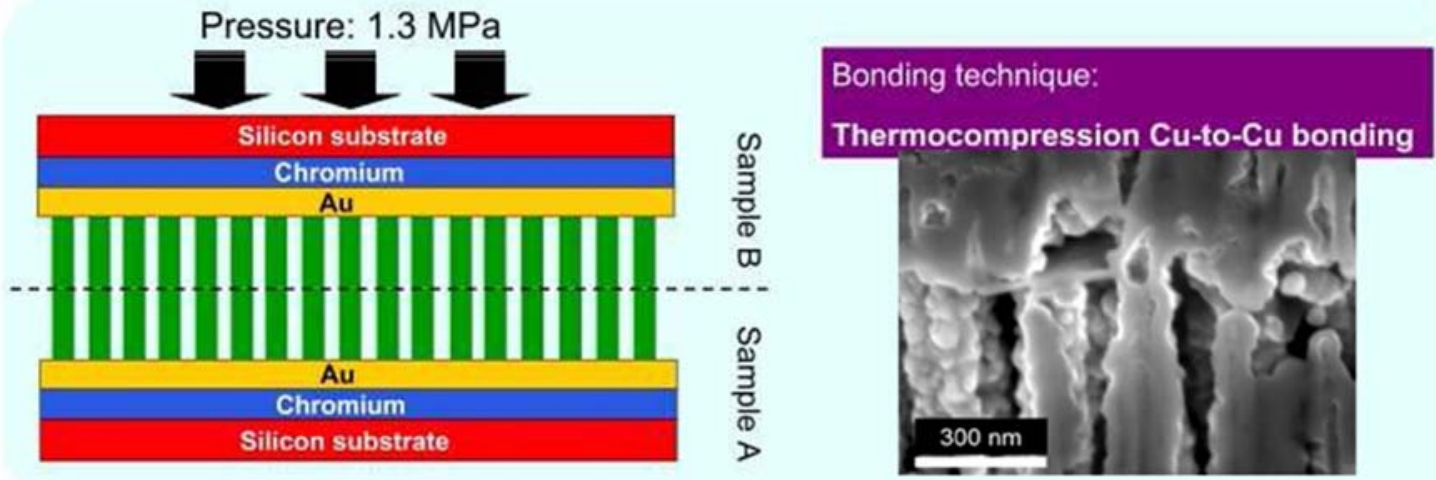

Fig. (8). Two pieces of 2D copper nanowire arrays face-to-face bonding at $300^{\circ} \mathrm{C}$ for 1 hour under a pressure of $1.3 \mathrm{Mpa}$. Reprinted with permission [61].

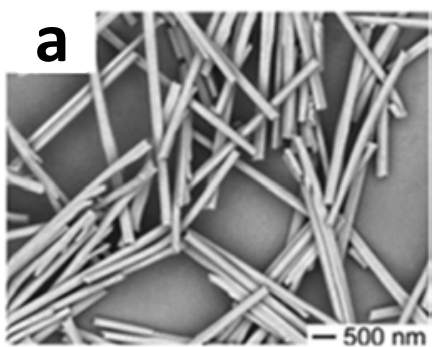

Un-bonded nanowires

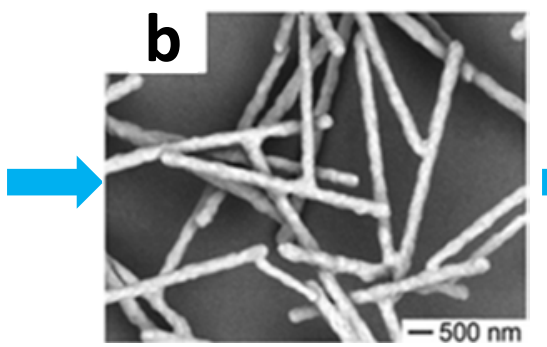

Bonded nanowires clusters

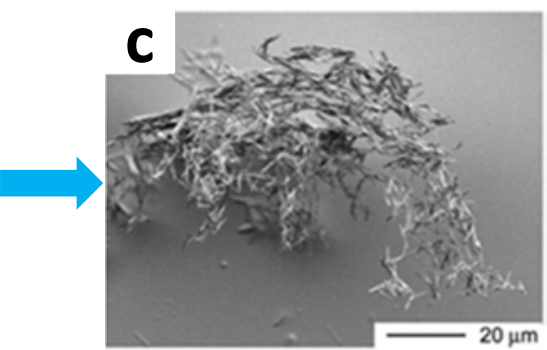

Bonded nanowire network

Fig. (9). Gold nanowires with $200 \mathrm{~nm}$ nominal diameter (a) before and (b) after diffusion bonding, and (c) SEM image of a 100- $\mu \mathrm{m}-\mathrm{scale} 3 \mathrm{D}$ Au NW network. Reprinted with permission [41].

reflow process usually follows a temperature programmed heating procedure in an oven with either air or nitrogen atmospheric environment. While in the "liquid reflow", the conventional gas phase environment is replaced by a liquid with high boiling point so that nanostructures are able to move freely and form the assembled structure by external forces. The result of massive nanowires joined together by nanosoldering is shown in Fig. (10). Fig. (10a) shows the multisegmented nanowires before "liquid reflow" where different segments can be distinguished by the contrast. Fig. (10b) presents the result from a "liquid reflow" process in which nanowires were well aligned and assembled into a network by soldered joints. Fig. (10c, d) show clearly the formation of solder joints only at the tip area of each nanowire. In all, nanowire soldering is a promising method for future device miniaturization and assembly, considering the intensive usage of soldering in the electronics industry.

\section{NANOWIRE-ELECTRODE/CONTACT PAD JOINING}

At the early stage of nanowire related researches, driven by the demand of nanowire characterization, many methods have been utilized to make nanowire-electrode contacts
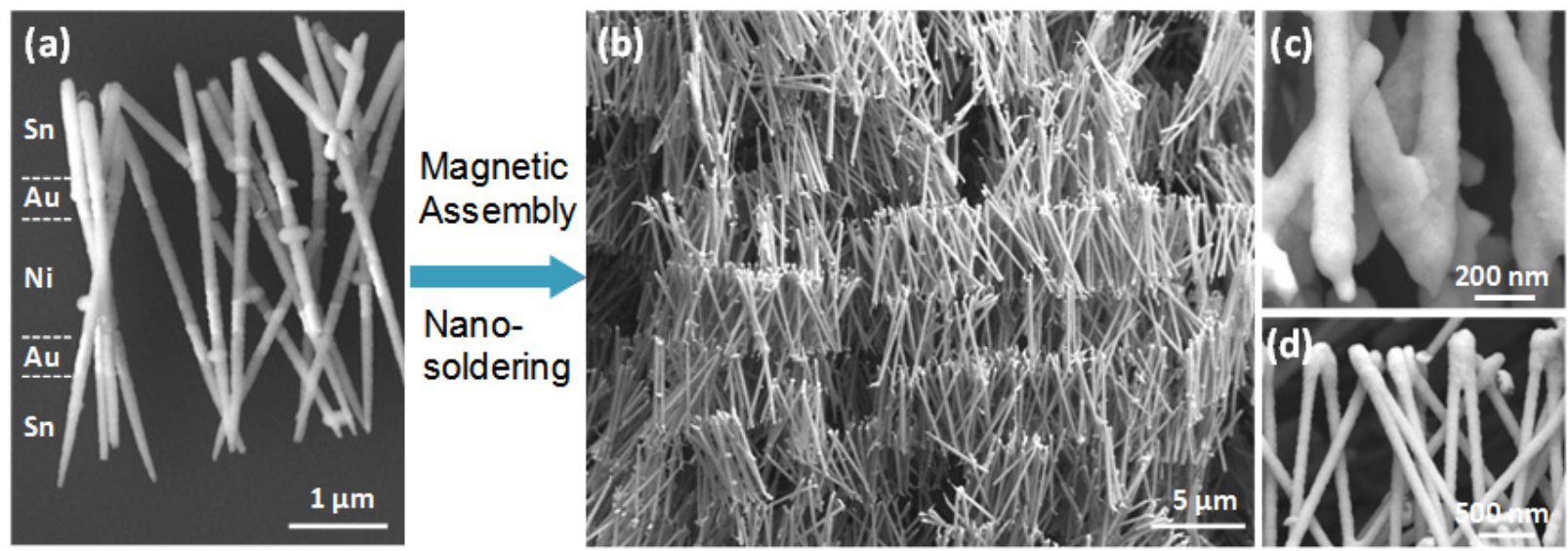

Fig. (10). SEM images of large-scale 3D soldered nanowire networks formed under magnetic assembly in a liquid reflow process inside an external magnetic field. (a) As fabricated nanosolder nanowires composed of $\mathrm{Sn}-\mathrm{Au}-\mathrm{Ni}-\mathrm{Au}-\mathrm{Sn}$ multi-segment. (b) Well aligned and soldered nanowire networks after the soldering; (c, d) Zoom in images of nanowires joining structures formed by reflowed nanosolders. 
regardless of the cost, complexity and efficiency. This is valuable for researchers to obtain the first hand data of the electrical, mechanical and thermal properties of nanowires. With the increasing interest on massive nanowire integration for electronic devices and real world applications, there is a need for alternative techniques which should be more affordable, efficient and less dependent on advanced equipments. In the following paragraphs, some of the currently available joining techniques will be reviewed, including soldering, fastener materials and coatings, and a novel technique called hot-pressing.

\subsection{Soldering}

In the previous discussion, multisegmented nanowires with solder materials caped on both ends have been proved to be able to efficiently join the nanowires. Combined with self assembly techniques such as magnetic, dielectrophoretic (DEP) assembly, this strategy could be extended to solder the nanocomponents with large sized contact pads [58, 76]. Soldering is very necessary because the contact condition of field force assembled nanowires with electrodes is very poor and needs enhancement. In most cases, the electrodes fabricated using current metallization methods have a high surface roughness comparing to the size of nanowires, and the real contact area is small. In other words, nanowire and electrode mainly have point-to-point contacts, leading to high contact resistance. During the solder reflow process, the solder materials liquefied and wetted the electrode surface, thus the contact condition dramatically improved. Fig. (11) shows the electrical resistance measured between two contact pads coated with three layers of $\mathrm{Ni}-\mathrm{Cu}$-solder $(\mathrm{Sn} / \mathrm{Pb})$ that were bridged with one $\mathrm{Au}-\mathrm{Ni}-\mathrm{Au}$ nanowire before, during and after the solder reflow. There was a huge contact resistance drop when the temperature reached $230{ }^{\circ} \mathrm{C}$ (above the melting point of $\mathrm{Sn} / \mathrm{Pb}$ solder) in a magnitude several hundred times lower than the original value. Besides the electrical contact, the mechanical strength also improved when solder materials solidified and held the nanowires on the contact pads [77]. The formation of solder joints is evident in Fig. (11) compared to the image of the same nanowire before solder reflow.

In another example, a pseudo-soldering method was developed and applied for the integration of tin or indium-tin oxide based nanowire sensor device [14]. The pure tin or indium-tin alloy nanowires were first positioned between interdigitated platinum electrodes using DEP method. Several critical parameters like frequency and amplitude of the applied electric field was investigated to tune up the assembly process. Fig. (12a, b) provide a comparison of nanowire assembly under non-optimized and optimized setting. Obviously, the yield of vertically (to the electrodes) orientated nanowires was low at frequency of $10 \mathrm{kHz}$ and amplitude of $0.1 \mathrm{~V}$, while a better result was observed using 6 $\mathrm{MHz}$ and $6 \mathrm{~V}$. Also, the concentration of nanowires assembled on the electrode substrate could be controlled to be either dense or dilute, as shown in Fig. (12c, d), respectively.

Then these nanowires were heated to the temperature about 10 degrees lower than the melting point of the metal or alloys in a nitrogen atmosphere. Since no oxidation was involved, the softening of the material improved the contact area condition along with possible diffusion between the nanowires and the metallic electrodes. Fig. (13a) shows a top-view of DEP assembled indium-tin alloy nanowires bridging interdigitated electrodes. The following electrical measurement results of the system resistance before and after pseudo-soldering were shown in Fig. $(\mathbf{1 3 b}, \mathbf{c})$ respectively.

Instead of attaching solder materials to the nanowire, Hangarter et al. galvanically plated a $1 \mu \mathrm{m}$ solder film $(60 \mathrm{Sn} 40 \mathrm{~Pb})$ on top of the nickel electrodes to perform the soldering [78]. After magnetic alignment, nanowires were placed on the pre-coated solder layer of the electrodes. Then the naowires were heated to $350{ }^{\circ} \mathrm{C}$ for 5 minutes for solder reflow and establishing better electrical contact.

\subsection{Fastener Materials and Coatings}

Fastener materials and coatings are often seen in mechanical joining systems. In nanoscale, a deposited thin film functions as the reinforcement on specific areas. This

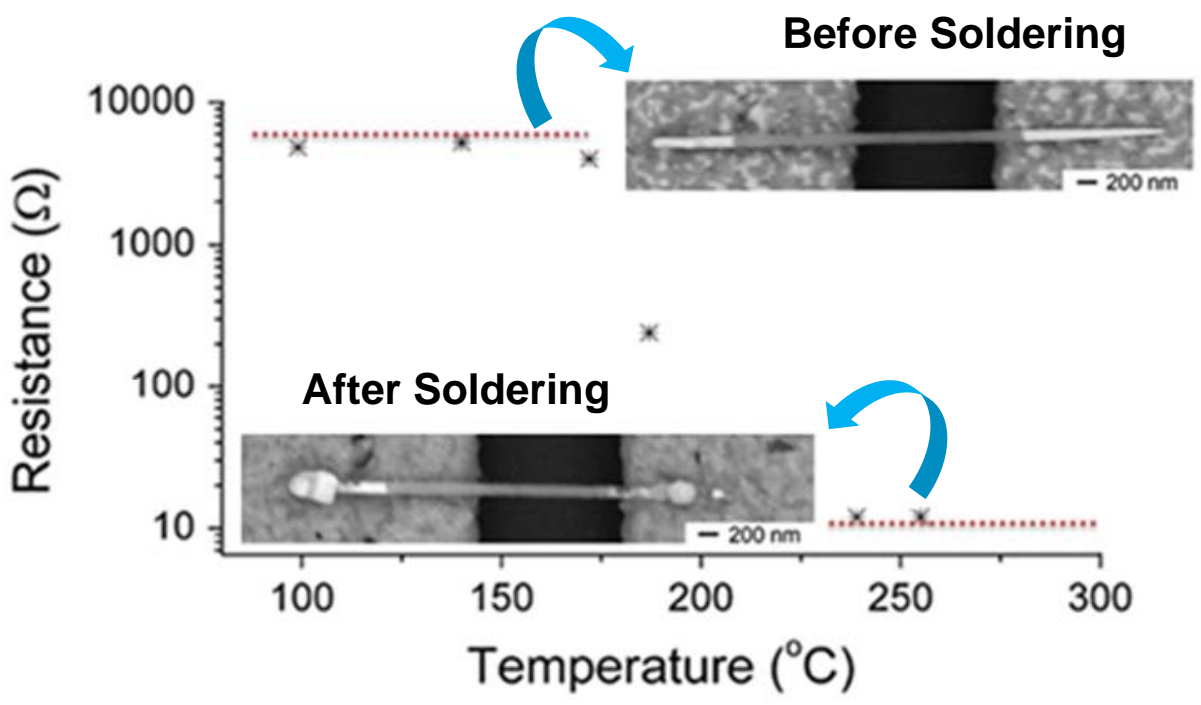

Fig. (11). Electrical resistance between nanowire-bridged contact pads measured during reflow showing a dramatic drop around $180{ }^{\circ} \mathrm{C}$. Insets are SEM images of 50-nm diameter nanowire before and after reflow [77]. 

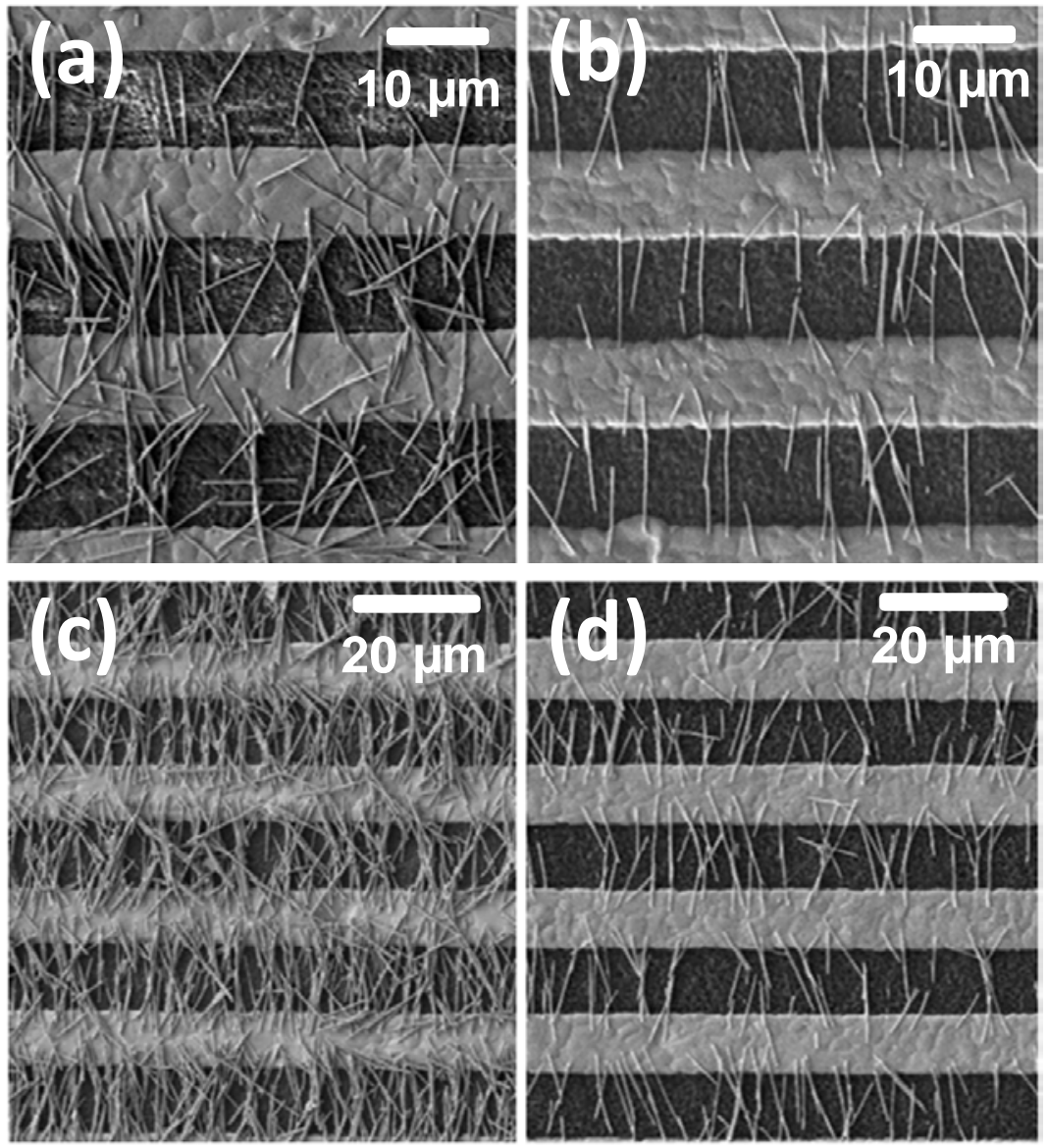

Fig. (12). SEM images of nanowires assembled on the electrode substrate using DEP method. (a) non-optimized setting; (b) optimized setting; (c) dense; (d) dilute.

could be achieved by either electron/ion beam induced deposition or electrodeposition [79, 80]. It is also possible to directly build the contact pads on top of the nanowires to form stable mechanical contact by different metallization methods $[81,82]$.

\subsubsection{Electron/Ion Beam Deposition}

Electron/ion beam is a very powerful tool that finds wide applications in more than image characterization and element analysis. The high spatial accuracy makes it promising for building structures in nanoscale. The adsorption of high energy electron/ion beam by the substrate causes the re-emit secondary electrons with a wide range of energies to decompose gaseous precursor molecules, leading to the deposition of non-volatile fragments onto the nearby area [83-85].

For enhancement purpose, the metal deposition takes place at the existing nanowire-contact pad overlapping area, improving the mechanical robustness and electrical connection. For example, Becker and colleagues successfully joined an AFM tip with a silicon nanowire for tip-enhanced Raman spectroscopy (TERS) probe by depositing metal material on the tip-nanowire interface [86, 87]. Another example displayed on Fig. (14), in which one Pd nanowire was manipulated to contact the MEMS device (Fig. 14a), and a layer of $\mathrm{Pt}$ was deposited on the overlapping areas of nanowire and contact pads (Fig. 14b); then this nanowire was tested by in situ transmission electron microscopy for stress-strain curve [88]. The result from the testing in Fig. (14c) not only showed that this nanowire has a Young's modulus of $99.4 \pm 6.6 \mathrm{GPa}$, which is higher than its bulk counterpart, but also indicated the high mechanical strength of the nanowire-contact pads joining.

However, in most cases electron/ion beam-induced deposition has been used to directly build electrodes/contact pads on top of randomly dispersed nanowires. Fig. (15) shows an image of typical four-probe electrodes built upon an individual tin oxide $\left(\mathrm{SnO}_{2}\right)$ nanowire for electrical conductivity studies [79]. Through electrical measurement, the contact resistances could be easily calculated for nanowires of different diameters with focus ion beam (FIB) deposited platinum microleads at room temperature. Furthermore, a portable microsensor device that features single tin dioxide nanowire as the sensing component has been fabricated using FIB deposited Pt electrodes [81].

\subsubsection{Photolithography}

Different from the 'direct write' style of electron/ion beam induced deposition, photolithography has to go through a series of processes, including photoresist (PR) coating, exposure, development, metallization, and lift-off. Since the electrode building process is carried out after the drop casting of nanowire sample on the substrate $[89,90]$, the sample may be damaged during soft/post baking, PR development or lift-off. Furthermore, if no alignment was conducted before exposure, the yield of effective nanowire- 

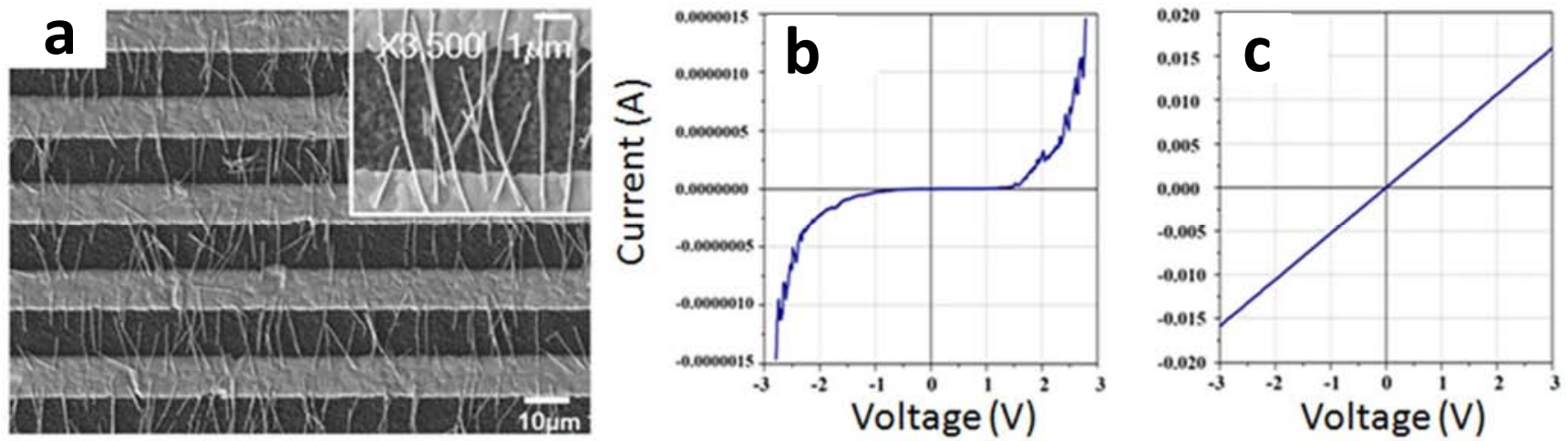

Fig. (13). A top-view of DEP assembled nanowires bridging interdigitated electrodes (a); Current-voltage characteristic curve of nanowireelectrode contacts before (b) and after (c) pseudo-soldering [14].
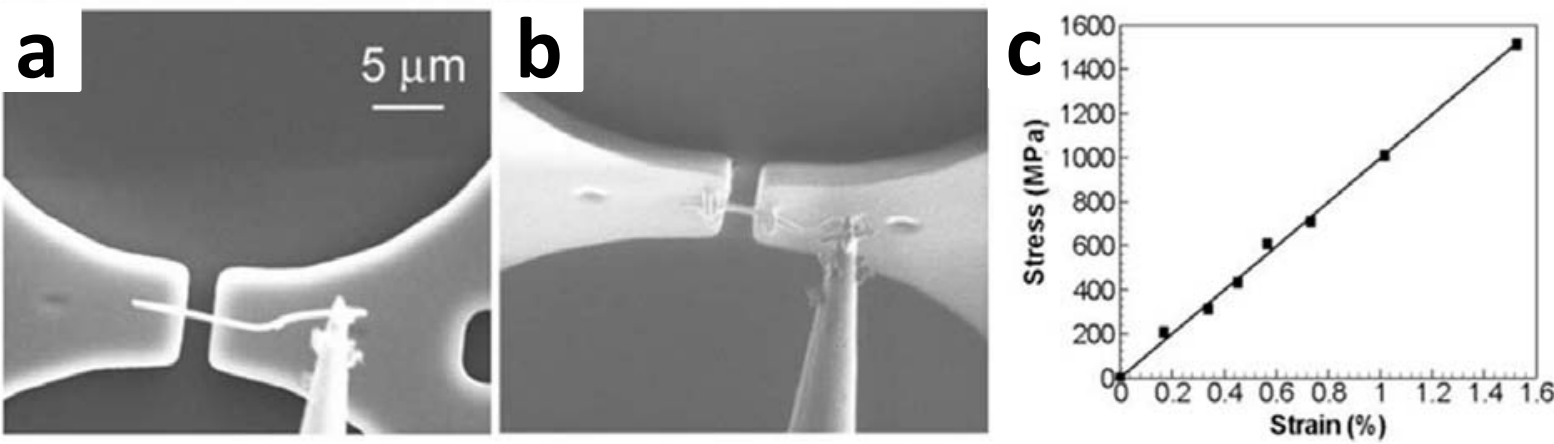

Fig. (14). Nanomanipulation procedure to mount a Pd nanowire in a MEMS device and the test results. (a) Approach and contact the nanowire to the MEMS device. (b) Deposit Pt layer on the two ends of the nanowire. (c) Stress-strain curve of a Pd nanowire exhibiting a Young's modulus of $99.4 \pm 6.6 \mathrm{GPa}$ and a strength higher than its bulk counterpart. Reprinted with permission [88].

electrodes contact would be very low. In Fig. (16), a Ge nanowire has been utilized as a channel with photolithographically fabricated source and drain electrodes to form a transistor [82]. In addition, this method only applies to single or few nanowires, and is not economical for high-rate, large scale manufacturing.

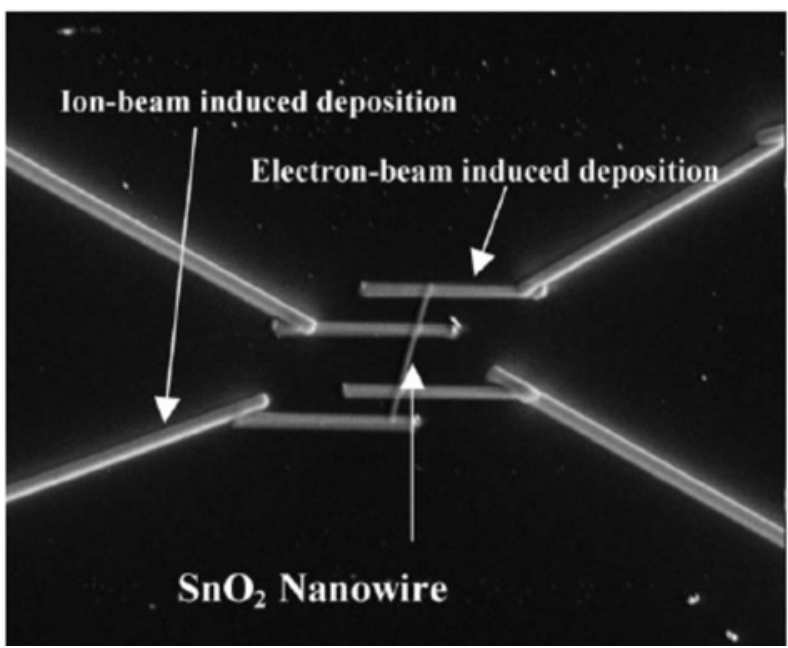

Fig. (15). $\mathrm{SnO}_{2}$ nanowire electrically contacted using FIB lithography technique. Both electron- and ion-beam induced deposition are indicated. Reprinted with permission [79]. (a)
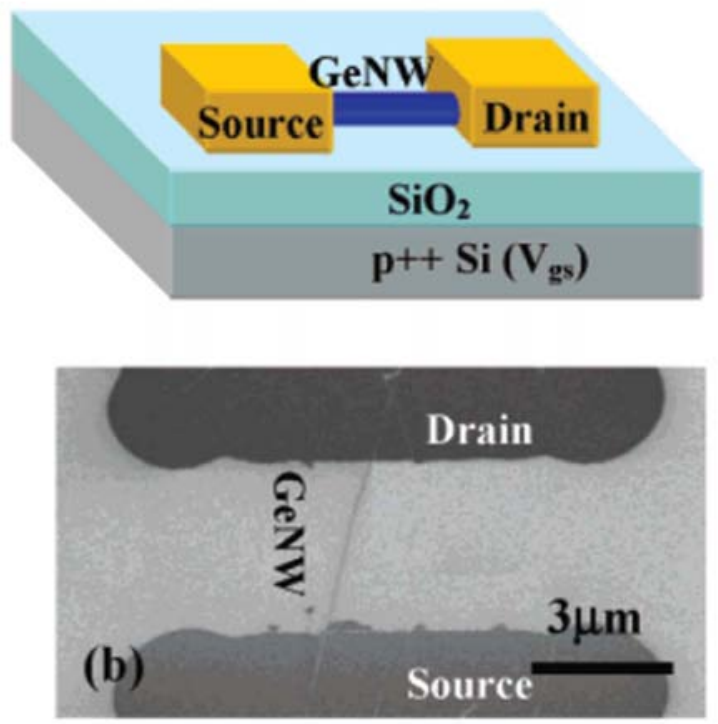

Fig. (16). (a) Schematic demonstration of a nanowire transistor fabricated by photolithography patterning. (b) SEM image of a single Ge nanowire transistor with source and drain electrode. Reprinted with permission [82].

One problem for the electron/ion beam induced deposition is that it is very difficult to fabricate complex structures due to the high expense and slow process. In order to compensate this weakness, sometimes the photolitho- 


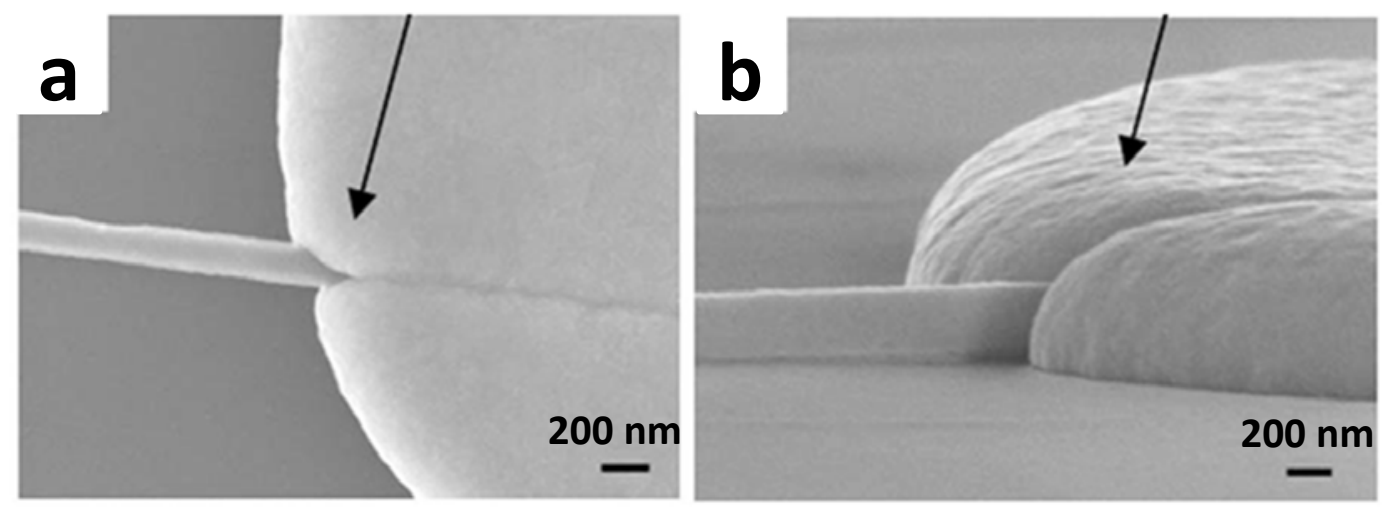

Fig. (17). High magnification (a) plane and (b) inclined views near one of the nanowire-electrode junctions after electrodeposition. Reprinted with permission [80].

graphically fabricated electrodes are used as the substrate for nanowire dispersion, and then the electron/ion beam induced deposition will only be performed on nanowires close to the pre-fabricated electrodes.

\subsubsection{Electrodeposition}

The surface thickening of electrodes could be achieved using electroless plating, an auto-catalytic, non-galvanic type of plating, or conventional electrolytic plating which requires external current/voltage applied to the system [73, 91]. Considering that electrodeposition may only occur in the liquid phase, and most electroless plating or electrolytic solutions are either acidic or basic, some metal oxides are inherently against this method, such as zinc oxide [92]. However, semiconductor [93] or conducting polymer nanowires [94] do not have such concerns. As shown in Fig. (17), after deposition, the silicon nanowire was embedded tightly in a thick layer of metal and both mechanical support and electrical contact problem were solved [80]. Although the deposition can be controlled by the reaction duration, lateral growth of deposited metal is inevitable. Thus this method cannot be used for structures with gap distance too small that can be easily covered by even a short period of metal growth.

\subsection{Hot-Pressing}

Besides conventional photolithography and ion/electron beam deposition, hot-pressing is another approach to solve the poor electrical contact issue occurred in most field force assembled nanowires with contact pads [95]. It outperforms the former two methods by its simple set-up, operation easiness and efficiency. The concept of hot-pressing is very straightforward. Once the nanowires were positioned by dielectrophoretic force with two ends sitting on the source and drain electrodes, a piece of silicon wafer was put on top of the structure. To avoid adherence of nanowires, the contact surface of this silicon wafer was coated with diamond like carbon (DLC) film. Then a hot-embossing apparatus was utilized to hot-press this sandwiched nanowire-electrode structure at $180^{\circ} \mathrm{C}$. A schematic diagram of this process is shown in Fig. (18a), and the SEM image of the nanowire-electrode interconnects after hot-pressing in Fig. (18b) shows that the nanowire was enchased into the aluminum electrode surface. The reported electrical measurement and transistor characteristics further proved the good contacts between nanowires and the electrodes.

\section{SUMMARY AND PERSPECTIVE}

This review has indicated that joining of nanowires could be achieved by various methods. Cold-welding and fusionwelding of nanowires are straightforward ways to cause coalescence of materials, and capable of providing strong and high purity joints by melting and merging. Fastener materials and coatings have been widely used to form nanowire-contact pad joining for characterization and device integration, normally for a limited number of nanowires. However, these two methods may heavily rely on highprecision manipulation tools and advanced in situ equipments to apply the current, heat or metal deposition. Nanoscale soldering, on the other hand, looks very promising to join nanowires in a versatile and low cost way by integrating solder materials into the nanowire. These low melting point metals will form nanoscale solder joints by a simple reflow process, which is already adapted in modern electronics industry. Besides those methods, the newly reported hot-pressing method is another creative and simple way to join nanowires with metal contact pads.

The future research work of nanowire joining should be focused on the stability, device reliability and scalability in conjugation with nanowire fabrication techniques and assembling methods. Several key problems such as reducing cost, improving efficiency and yield, and realization of massive integration need to be addressed. Even though there are challenges ahead, there are plenty of great opportunities for nanowire joining techniques to be adopted in nanoelectronics and devices that can benefit our life and society.

\section{ACKNOWLEDGEMENTS}

We are grateful for the financial support from the following funding sources: the faculty start-up fund from the University, NSF NSEC Center for High-rate Nanomanufacturing $(\mathrm{CHN})$, the Sustainability Research Fellow Program and Academic Research Program from the Toxics Use Reduction Institute (TURI), and 3M Non-tenured Faculty Grant. X. Li's work is supported by the National Science Foundation (grant number ECCS-0731125). 


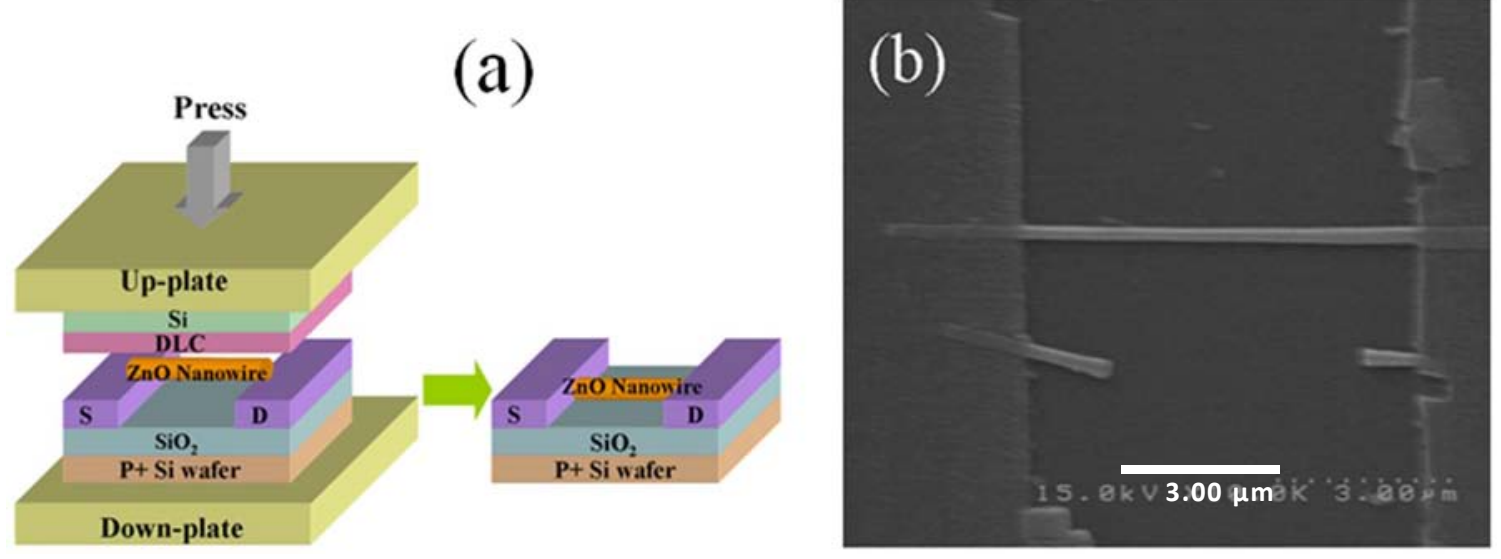

Fig. (18). (a) A schematic diagram of hot-pressing. (b) SEM image of nanowire-electrode junctions after hot-pressing. Reprinted with permission [95].

\section{REFERENCES}

[1] Iijima S. Helical microtubules of graphitic carbon. Nature 1991; 354(6348): 56-8.

[2] Chang P, Fan Z, Chien C, Stichtenoth D, Ronning C, Lu J. Highperformance $\mathrm{ZnO}$ nanowire field effect transistors. Appl Phys Lett 2006; 89: 133113

[3] Patolsky F, Zheng G, Lieber C. Nanowire-based biosensors. Anal Chem 2006; 78(13): 4260-9.

[4] Zhang J, Zhang L, Peng X, Wang X. Vapor-solid growth route to single-crystalline indium nitride nanowires. J Mater Chem 2002; 12(4): 802-4.

[5] Yang P, Yan H, Mao S, et al. Controlled growth of $\mathrm{ZnO}$ nanowires and their optical properties. Adv Funct Mater 2002; 12(5): 323-31.

[6] Wang F, Dong A, Sun J, Tang R, Yu H, Buhro W. Solution- liquidsolid growth of semiconductor nanowires. Inorg Chem 2006; 45(19): 7511-21.

[7] Sawicka K, Prasad A, Gouma P. Metal oxide nanowires for use in chemical sensing applications. Sens Lett 2005; 1(4): 31-5.

[8] Prieto A, Sander M, Martin-Gonzalez M, Gronsky R, Sands T, Stacy A. Electrodeposition of ordered Bi2Te3 nanowire arrays. J Am Chem Soc 2001; 123(29): 7160-1.

[9] Yin A, Li J, Jian W, Bennett A, Xu J. Fabrication of highly ordered metallic nanowire arrays by electrodeposition. Appl Phys Lett 2001; 79: 1039 .

[10] Chen J, Zhang F, Wang J, et al. CuO nanowires synthesized by thermal oxidation route. J Alloy Comp 2008; 454(1-2): 268-73.

[11] Kaur M, Muthe K, Despande S, et al. Growth and branching of $\mathrm{CuO}$ nanowires by thermal oxidation of copper. J Cryst Growth 2006; 289(2): 670-5.

[12] Basnar B, Willner I. Dip-pen-nanolithographic patterning of metallic, semiconductor, and metal oxide nanostructures on surfaces. Small 2009; 5(1): 28-44.

[13] Hung C, Gui J, Switzer J. Scanning probe nanolithography of conducting metal oxides. Appl Phys Lett 1997; 71: 1637.

[14] Li X, Chin E, Sun H, Kurup P, Gu Z. Fabrication and Integration of Metal Oxide Nanowire Sensors Using Dielectrophoretic Assembly and Improved Post-Assembly Processing. Sens Actuators B 2010; 148(2): 404-12.

[15] Fan H, Werner P, Zacharias M. Semiconductor nanowires: from self organization to patterned growth. Small 2006; 2(6): 700-17.

[16] Mukherjee S, Li X, Gao F, Gu Z. An Efficient silver etchant for the fabrication of active nanowires using anodized aluminum oxide templates. Electrochem Solid-State Lett 2010; 13(7): D50.

[17] Briseno A, Mannsfeld S, Jenekhe S, Bao Z, Xia Y. Introducing organic nanowire transistors. Mater Today 2008; 11(4): 38-47.

[18] Lu W, Xie P, Lieber C. Nanowire transistor performance limits and applications. IEEE Trans on Electr Device 2008; 55(11): 2859-76.

[19] Liu H, Kameoka J, Czaplewski D, Craighead H. Polymeric nanowire chemical sensor. Nano Lett 2004; 4(4): 671-5.

[20] Fan Z, Lu J. Gate-refreshable nanowire chemical sensors. Appl Phys Lett 2005; 86: 123510.
[21] Agarwal R, Lieber C. Semiconductor nanowires: optics and optoelectronics. Appl Phys A: Mater Sci Process 2006; 85(3): 20915.

[22] Choi S, Kim J, Jung J, Yoon E, Kim W. Pt nanowires prepared via a polymer template method: Its promise toward high Pt-loaded electrocatalysts for methanol oxidation. Electrochim Acta 2008; 53(19): 5804-11.

[23] Kostowskyj M, Gilliam R, Kirk D, Thorpe S. Silver nanowire catalysts for alkaline fuel cells. Int J Hydrog Energy 2008; 33(20): 5773-8.

[24] Chan C, Peng H, Liu G, et al. High-performance lithium battery anodes using silicon nanowires. Nat Nanotechnol 2007; 3(1): 31-5. Chan C, Connor S, Yang Y, Hsu C, Huggins R, Cui Y. Nanowire batteries for next generation electronics; Electron Devices Meeting, IEEE Int 2008.

[26] Law M, Greene L, Johnson J, Saykally R, Yang P. Nanowire dyesensitized solar cells. Nat Mater 2005; 4(6): 455-9.

[27] Tsakalakos L, Balch J, Fronheiser J, Korevaar B, Sulima O, Rand J. Silicon nanowire solar cells. Appl Phys Lett 2007; 91: 233117.

[28] Yan R, Gargas D, Yang P. Nanowire photonics. Nat Photon 2009; 3(10): 569-76.

[29] Pauzauskie P, Yang P. Nanowire photonics. Mater Today 2006; 9(10): 36-45

[30] Li Y, Qian F, Xiang J, Lieber C. Nanowire electronic and optoelectronic devices. Mater Today 2006; 9(10): 18-27.

[31] Huang X, Zorman C, Mehregany M, Roukes M. Nanoelectromechanical systems: Nanodevice motion at microwave frequencies. Nature 2003; 421(6922): 496.

[32] Ekinci K, Roukes M. Nanoelectromechanical systems. Rev Scientific Instrum 2005; 76: 061101

[33] Cheng C, Gonela R, Gu Q, Haynie D. Self-assembly of metallic nanowires from aqueous solution. Nano Lett 2005; 5(1): 175-8.

[34] Gates B. Self-assembly: Nanowires find their place. Nat Nanotechnol 2010; 5(7): 484-5.

[35] Wang M, Gates B. Directed assembly of nanowires. Mater Today 2009; 12(5): 34-43.

[36] Liang L, Liu J, Windisch Jr C, Exarhos G, Lin Y. Direct assembly of large arrays of oriented conducting polymer nanowires. Angew Chem Int Ed 2002; 41(19): 3665-8.

[37] Javey A, Nam S, Friedman R, Yan H, Lieber C. Layer-by-layer assembly of nanowires for three-dimensional, multifunctional electronics. Nano Lett 2007; 7(3): 773-7.

[38] Rajurkar K, Levy G, Malshe A, et al. Micro and nano machining by electro-physical and chemical processes. CIRP AnnalsManufact Technol 2006; 55(2): 643-66.

[39] Shinno H, Yoshioka H, Taniguchi K. A newly developed linear motor-driven aerostatic XY planar motion table system for nanomachining. CIRP Annals-Manufact Technol 2007; 56(1): 369-72.

[40] Lu Y, Huang J, Wang C, Sun S, Lou J. Cold welding of ultrathin gold nanowires. Nat Nanotechnol 2010; 5(3): 218-24.

[41] Gu Z, Ye H, Bernfeld A, Livi K, Gracias D. Three-dimensional electrically interconnected nanowire networks formed by diffusion bonding. Langmuir 2007; 23(3): 979-82. 
[42] Ferguson GS, Chaudhury MK, Sigal GB, Whitesides GM. Contact Adhesion of Thin Gold Films on Elastomeric Supports: Cold Welding Under Ambient Conditions. Science 1991; 253(5021): 776-8.

[43] Freitas R, Healy T, Long J, Eds. Advanced automation for space missions. Morgan Kaufmann Publishers Inc 1981.

[44] Kizuka T. Atomic process of point contact in gold studied by timeresolved high-resolution transmission electron microscopy. Phys Rev Lett 1998; 81(20): 4448-51.

[45] van Huis M, Kunneman L, Overgaag K, et al. Low-temperature nanocrystal unification through rotations and relaxations probed by in situ transmission electron microscopy. Nano Lett 2008; 8(11): 3959-63.

[46] Cho K, Talapin D, Gaschler W, Murray C. Designing PbSe nanowires and nanorings through oriented attachment of nanoparticles. J Am Chem Soc 2005; 127(19): 7140-7.

[47] Tohmyoh H, Imaizumi T, Hayashi H, Saka M. Welding of Pt nanowires by Joule heating. Scripta Materialia 2007; 57(10): 953-6.

[48] Fukui S, Tohmyoh H, Saka M, Eds. 3D welding of ultrathin Pt wires by Joule heating. Electronic System-Integration Technology Conference (ESTC), September 2010. Berlin, Germany: IEEE; 2011.

[49] Takeda S, Koto K, Iijima S, Ichihashi T. Nanoholes on silicon surface created by electron irradiation under ultrahigh vacuum environment. Phys Rev Lett 1997; 79(16): 2994-7.

[50] Bysakh S, Shimojo M, Mitsuishi K, Furuya K. Mechanisms of nano-hole drilling due to nano-probe intense electron beam irradiation on a stainless steel. J Vac Sci Technol B 2004; 22: 2620.

[51] Storm A, Chen J, Ling X, Zandbergen H, Dekker C. Fabrication of solid-state nanopores with single-nanometre precision. Nat Mater 2003; 2(8): 537-40.

[52] Yokota T, Murayama M, Howe J. In situ transmission-electronmicroscopy investigation of melting in submicron Al-Si alloy particles under electron-beam irradiation. Phys Rev Lett 2003; 91(26): 265504.

[53] Takeda S, Yamasaki J. Amorphization in silicon by electron irradiation. Phys Rev Lett 1999; 83(2): 320-3.

[54] Xu S, Tian M, Wang J, Xu J, Redwing J, Chan M. Nanometer-scale modification and welding of silicon and metallic nanowires with a high-intensity electron beam. Small 2005; 1(12): 1221-9.

[55] Wu Y, Yang P. Melting and welding semiconductor nanowires in nanotubes. Adv Mater 2001; 13(7): 520-3.

[56] Peng Y, Cullis T, Inkson B. Bottom-up nanoconstruction by the welding of individual metallic nanoobjects using nanoscale solder. Nano Lett 2008; 9(1): 91-6.

[57] Peng Y, Cullis A, Inkson B. Nanoconstruction by welding individual metallic nanowires together using nanoscale solder. J Phys: Conf Ser 2010; 241: 012083.

[58] Gu Z, Ye H, Gracias D, Gracias D. The bonding of nanowire assemblies using adhesive and solder. J Miner Met Mater Soc 2005; 57(12): 60-4.

[59] Gu Z, Ye H, Smirnova D, Small D, Gracias D. Reflow and electrical characteristics of nanoscale solder. Small 2006; 2(2): 225-9.

[60] Gu Z, Chen Y, Gracias D. Surface tension driven self-assembly of bundles and networks of $200 \mathrm{~nm}$ diameter rods using a polymerizable adhesive. Langmuir 2004; 20(26): 11308-11.

[61] Ng M. Low Temperature Bonding via Copper Nanowires for 3D Integrated circuits. Mater Res Soc Symp Proc 2010; 1249: F04-21.

[62] Thomson J, Lawson G, O'Brien P, et al. Flash Nano-Welding: Investigation and Control of the Photothermal Response of Ultrathin Bismuth Sulfide Nanowire Films. Adv Mater 2010; 22(39): 4395-4400.

[63] Ajayan P, Terrones M, de la Guardia A, et al. Nanotubes in a Flash--Ignition and Reconstruction. Science 2002; 296(5568): 705.

[64] Kang B, Dai Y, Chang S, Chen D. Explosion of single-walled carbon nanotubes in suspension induced by a large photoacoustic effect. Carbon 2008; 46(6): 978-81.

[65] Lien D, Kuo H, Hsu W. Segmentation of single-walled carbon nanotubes by camera flash. Appl Phys Lett 2006; 88: 093113.

[66] Tseng S, Tai N, Hsu W, et al. Ignition of carbon nanotubes using a photoflash. Carbon 2007; 45(5): 958-64.
[67] Cui Q, Gao F, Mukherjee S, Gu Z. Joining and interconnect formation of nanowires and carbon nanotubes for nanoelectronics and nanosystems. Small 2009; 5(11): 1246-57.

[68] Mosley D, Chow B, Jacobson J. Solid-state bonding technique for template-stripped ultraflat gold substrates. Langmuir 2006; 22(6): 2437-40.

[69] Ornellas DL, Catalano E. Diffusion bonding of gold to gold. Rev Sci Instrum 1974; 45(7): 955.

[70] Kazakov N, Kuznetsov B. Diffusion bonding of materials. Oxford: Pergamon Press 1985.

[71] Tu K, Gusak A, Li M. Physics and materials challenges for leadfree solders. J Appl Phys 2003; 93: 1335.

[72] Suganuma K. Advances in lead-free electronics soldering. Curr Opin Solid State Mater Sci 2001; 5(1): 55-64.

[73] Liebau M, Unger E, Duesberg G, et al. Contact improvement of carbon nanotubes via electroless nickel deposition. Appl Phys A 2003; 77(6): 731-4.

[74] Gao F, Mukherjee S, Cui Q, Gu Z. Synthesis, characterization, and thermal properties of nanoscale lead-free solders on multisegmented metal nanowires. J Phys Chem C 2009; 113(22): 9546-52.

[75] Gao F, Gu Z. Nano-soldering of magnetically aligned threedimensional nanowire networks. Nanotechnology 2010; 21: 115604.

[76] Ye H, Gu Z, Yu T, Bernfeld A, Leong T, Gracias D. Forming low resistance nano-scale contacts using solder reflow: Proceeding of 5th IEEE conference on Nanotechnology. Nagoya, Japan 2005.

[77] Ye H, Gu Z, Yu T, Gracias D. Integrating nanowires with substrates using directed assembly and nanoscale soldering. IEEE Trans Nanotechnol 2006; 5(1): 62-6.

[78] Hangarter C, Myung N. Magnetic alignment of nanowires. Chem Mater 2005; 17(6): 1320-4.

[79] Hernandez-Ramirez F, Rodriguez J, Casals O, et al. Characterization of metal-oxide nanosensors fabricated with focused ion beam (FIB). Sens Actuators B 2006; 118(2): 198-203.

[80] Ingole S, Aella P, Hearne S, Picraux S. Directed assembly of nanowire contacts using electrodeposition. Appl Phys Lett 2009; 91(3): 033106.

[81] Hernandez-Ramirez F, Prades J, Tarancon A, et al. Portable microsensors based on individual $\mathrm{SnO} 2$ nanowires. Nanotechnology 2007; 18: 495501.

[82] Wang D, Chang Y, Wang Q, et al. Surface chemistry and electrical properties of germanium nanowires. J Am Chem Soc 2004; 126(37): 11602-11.

[83] Randolph S, Fowlkes J, Rack P. Focused, nanoscale electron-beaminduced deposition and etching. Crit Rev Solid State Mater Sci 2006; 31(3): 55-89.

[84] Furuya K. Nanofabrication by advanced electron microscopy using intense and focused beam. Sci Technol Adv Mater 2008; 9: 014110.

[85] Song M, Furuya K. Fabrication and characterization of nanostructures on insulator substrates by electron-beam-induced deposition. Sci Technol Adv Mater 2008; 9: 023002.

[86] Becker M, Sivakov V, Gosele U, et al. Nanowires enabling signalenhanced nanoscale Raman spectroscopy. Small 2008; 4(4): 398404.

[87] Christiansen S, Becker M, Fahlbusch S, et al. Signal enhancement in nano-Raman spectroscopy by gold caps on silicon nanowires obtained by vapour-liquid-solid growth. Nanotechnology 2007; 18: 035503 .

[88] Zhu Y, Espinosa H. An electromechanical material testing system for in situ electron microscopy and applications. Proc Nat Acad Sci USA 2005; 102(41): 14503.

[89] Keem K, Kang J, Yoon C, et al. A fabrication technique for topgate $\mathrm{ZnO}$ nanowire field-effect transistors by a photolithography process. Microelectron Eng 2007; 84(5-8): 1622-6.

[90] Park J, Nalwa K, Leung W, Constant K, Chaudhary S, Ho K. Fabrication of metallic nanowires and nanoribbons using laser interference lithography and shadow lithography. Nanotechnology 2010; 21(21): 215301 . 
[91] Seidel R, Liebau M, Duesberg G, et al. In situ contacted singlewalled carbon nanotubes and contact improvement by electroless deposition. Nano Lett 2003; 3(7): 965-8.

[92] Yoshida T. Leaching of zinc oxide in acidic solution. Mater Trans 2003; 44(12): 2489-93.

[93] Suh D, Lee S, Hyung J, Kim T, Lee S. Multiple $\mathrm{ZnO}$ nanowires field-effect transistors. J Phys Chem C 2008; 112(4): 1276-81.
[94] Hangarter C, Bangar M, Hernandez S, et al. Maskless electrodeposited contact for conducting polymer nanowires. Appl Phys Lett 2008; 92: 073104.

[95] Chang Y, Hong F. The fabrication of $\mathrm{ZnO}$ nanowire field-effect transistors combining dielectrophoresis and hot-pressing. Nanotechnology 2009; 20: 235202.

Received: November 1, 2010

(C) Li et al.; Licensee Bentham Open.

This is an open access article licensed under the terms of the Creative Commons Attribution Non-Commercial License (http://creativecommons.org/licenses/ by$\mathrm{nc} / 3.0 /$ ) which permits unrestricted, non-commercial use, distribution and reproduction in any medium, provided the work is properly cited. 